\title{
Sequential Double Bridging to Transplant with Diversified Anti-PD1 Monoclonal Antibodies Retreatment in Relapsed Hodgkin Lymphoma: A Case Report
}

\author{
Cinzia Pellegrini ${ }^{1}$, Beatrice Casadei ${ }^{1}$, Claudia Cellini ${ }^{2}$, Lisa Argnani ${ }^{1}$, Michele Cavo $^{1}$ and Pier Luigi Zinzani* \\ ${ }^{1}$ Institute of Hematology "L. e. A. Seràgnoli", University of Bologna, Bologna, Italy
}

${ }^{2}$ Unit of Hematology, Santa Maria Delle Croci Hospital, Ravenna, Italy

\begin{abstract}
Background: Nivolumab and pembrolizumab are the first two US FDA-approved monoclonal antibodies targeting PD-1 for Hodgkin lymphoma $(\mathrm{HL})$ and provide promising results in the relapsed/refractory setting (HL patients relapsing after autologous stem cell transplantation [ASCT], or with chemorefractory disease and/ or ineligible for ASCT). An interesting area of ongoing research is the possibility of combining different immune checkpoint inhibitors given concomitantly or sequentially in the attempt to maximize the patient responsiveness.

Case report: A heavily pre-treated young woman affected by $\mathrm{HL}$, after several attempts, obtained a complete response after ASCT thanks to the bridge with pembrolizumab. After relapse, the patient achieved again a complete response with nivolumab, bridging her to allotransplant. The patient is still in response to a year since the transplant.

Discussion and Conclusion: This is the first report which witnesses the safety and the antitumor activity of interchangeable anti-PD1 monoclonal antibodies administered as a retreatment option and as a bridge to allotransplant in a patient who previously got an objective response to another anti-PD1 which brought her to autologous transplant. Retreatment with anti-PD1 monoclonal antibodies could be considered in therapeutic algorithm of relapsed/refractory HL.
\end{abstract}

Keywords: Hodgkin lymphoma; Anti-PD1 monoclonal antibodies; Autologous stem cell transplant; Allogeneic transplantation; Retreatment

\section{Introduction}

Hodgkin lymphoma (HL) is the most common lymphoma affecting young population. Around $90 \%$ of early stage $\mathrm{HL}$ and $70 \%$ of advanced stage patients are cured with conventional chemotherapy, while $25 \%$ of patients experiences a relapse or results refractory to first line treatment. The latter subset of patients is a high priority for research and drug innovation. For patients with progression after first line therapy, the standard of care is a second line chemotherapy followed by autologous stem cell transplant (ASCT), with a $50 \%$ rate of cure. In this setting, the most favorable prognostic factor is achieving a complete remission (CR) before ASCT [1]. For HL patients relapsing after ASCT, or with chemorefractory disease and/or ineligible for ASCT, there are several new agents such as brentuximab vedotin (BV), bendamustine, nivolumab and pembrolizumab that may improve their poor prognosis [2-5]. In this setting allogeneic stem cell transplantation (alloSCT) is considered as a therapeutic option in young patients with a suitable donor and chemo-sensitive disease [6].

Recent advances in the understanding of HL pathogenesis, interaction with tumor microenvironment and immune-escape mechanism have led to the identification of new therapeutic targets. Malignant Reed-Sternberg (RS) cells, which characterize HL, aberrantly express programmed death-1 (PD1) ligand and evade antitumor immune response by engaging programmed death-1 on immune effector cells $[2,7,8]$. Pembrolizumab and nivolumab are programmed death-1 blockade (anti-PD1) monoclonal antibodies (mAb) that restore immunity against Hodgkin RS cells. Anti-PD1 have recently demonstrated high (60\% to $80 \%)$ response rates in relapsed/refractory HL with manageable toxicities [9-11]. For patients who responded to anti-PD1 and then relapsed, the choice ranges from aggressive regimens that enable alloSCT to palliative care for improving patient's quality of life. Here, we report on the first patient to our knowledge successfully re-treated with an anti-PD1 mAb prior to alloSCT.

\section{Case Report}

In April 2014, a 21-year-old woman was diagnosed with classical HL involving lymphadenopathies both above and below the diaphragm, corresponding to a stage III B (pruritus). From April 2014 to October 2014 the patient received 6 cycles ABVD (adriamycin, bleomicine, dacarbazine, vinblastine). Final examination by $18 \mathrm{~F}$-fluorodeoxyglucose (FDG)-positron emission tomography (PET) and computed tomography (CT) demonstrated a residual metabolically active paramediastinal lymphnodes. Subsequently, the patient received two cycles of IGEV (ifosfamide, gemcitabine, epirubicin and vinorelbine) regimen with successfully harvest of $20.4 \times 10^{6} \mathrm{CD} 34+$ stem cells $/ \mathrm{kg}$ after second cycle; the PET showed a progressive disease (PD), with increase of lymphadenopathies both above and below the diaphragm and appearance of multiple skeletal lesions. Due to symptomatic disease the patient was treated with six cycles of BV. Response assessment showed multifocal PD, which was histologically confirmed by laterocervical lymphadenectomy. The patient was treated with further therapy, namely bendamustine at $90 \mathrm{mg} / \mathrm{m}^{2}$ on days 1 and 2 in a 28 -

*Corresponding author: Pier Luigi Zinzani, Institute of Hematology "L. e. A. Seràgnoli", University of Bologna, Bologna, Italy, Tel: +390512143680; Fax: +390512144037; E-mail: pierluigi.zinzani@unibo.it

Received May 25, 2018; Accepted June 18, 2018; Published June 20, 2018

Citation: Pellegrini C, Casadei B, Cellini C, Argnani L, Cavo M, et al. (2018) Sequential Double Bridging to Transplant with Diversified Anti-PD1 Monoclonal Antibodies Retreatment in Relapsed Hodgkin Lymphoma: A Case Report. J Cancer Sci Ther 10: 149-151. doi: 10.4172/1948-5956.1000534

Copyright: () 2018 Pellegrini C, et al. This is an open-access article distributed under the terms of the Creative Commons Attribution License, which permits unrestricted use, distribution, and reproduction in any medium, provided the original author and source are credited. 
Citation: Pellegrini C, Casadei B, Cellini C, Argnani L, Cavo M, et al. (2018) Sequential Double Bridging to Transplant with Diversified Anti-PD1 Monoclonal Antibodies Retreatment in Relapsed Hodgkin Lymphoma: A Case Report. J Cancer Sci Ther 10: 149-151. doi: 10.4172/19485956.1000534

day cycle schedule. She completed three courses of therapy, achieving a stable disease. In September 2015 the patient was enrolled in a phase II clinical trial and was treated with pembrolizumab at the dosage of 200 mg every 3 weeks with the aim to obtain a complete remission (CR) before being treated with ASCT. After 6 cycles of therapy the re-staging showed a CR and within one month the patient underwent ASCT. The chosen conditioning therapy was the BEAM (carmustine, etoposide, araC, melphalan) regimen. In September 2016, after six months from ASCT the patient had a recurrence of B symptoms (fever and pruritus). Disease relapse was documented by a routine follow-up PET witch showed multiple hypermetabolic lymph nodes both above and below the diaphragm and multiple skeletal lesions. At this point, a search for a matched unrelated donor for alloSCT was started.

Considering the disease chemorefractoriness but even the responsiveness to the anti-PD1 pembrolizumab, in October 2016 we decided to initiate an off-label therapy with another anti-PD1 mAb available in Italy, namely nivolumab at $3 \mathrm{mg} / \mathrm{kg}$ every 2 weeks, even in absence of retreatment clinical data. Treatment was well tolerated, B symptoms disappeared and patient clinical condition progressively improved over the following cycles. PET after 8 cycles showed a complete normalization of multiple hypermetabolic lymph nodes and skeletal lesions but also showed a new right inguinal lesion with SUV of 4.5. The CT scan (performed to check PET result) displayed small right inguinal lymph nodes hence it didn't confirm the presence of active disease. One month after the last course of nivolumab, in February 2017, following conditioning with cyclophosphamide (30 mg/kg), fludarabine (30 mg/ $\left.\mathrm{m}^{2}\right)$, thiotepa $(5 \mathrm{mg} / \mathrm{kg})$ and antithymocyte globulin $(6 \mathrm{mg} / \mathrm{kg})$, the patient underwent transplant from an HLA-matched unrelated donor. The graft versus host disease (GVHD) prophylaxis was characterized by methotrexate $\left(10 \mathrm{mg} / \mathrm{m}^{2}\right)$ and cyclosporine $1 \mathrm{mg} / \mathrm{kg}$ from day -6 to -2 and $2 \mathrm{mg} / \mathrm{kg}$ from day -1 . There was no development of acute or chronic post-transplant GVHD. The PET and CT re-staging after 3, 6, 9 and 12 months from allo-SCT exhibited a CR status.

\section{Discussion}

In spite of generally favorable prognosis of $\mathrm{HL}$, the prognosis of the primary refractory patients is poor. AlloSCT with reduced intensity conditioning, based on graft versus lymphoma, is a real chance to cure HL [6]. The most favorable prognostic factor is the disease control before transplant, with the highest rate of cure associated to negative PET [12].

PD1 signaling regulates immune response by decreasing T-cell activation and suppressing $\mathrm{T}$-cell proliferation. In general, many clinical trials on anti-PD1 mAbs have demonstrated interesting results in lymphoma patients with chemorefractory disease. In particular, HL have a unique biologic dependence on the PD1 pathway, because Hodgkin RS cells escape immune surveillance through a genetic alteration in chromosome 9p24.1, that leads to an overexpression of the PD1 ligands [12]. Results from phase 2 pivotal trial of single agent nivolumab $(3 \mathrm{mg} / \mathrm{kg})$ administered every 2 weeks demonstrated an objective response rate (ORR) of $87 \%(17 \% \mathrm{CR})$ in relapsed/refractory HL patients and a manageable profile of toxicity. Recent reports have confirmed a durable response [13]. In a second clinical trial with another anti-PD1 antibody, pembrolizumab, patients received therapy at the dose of $10 \mathrm{mg} / \mathrm{kg}$ every 2 weeks. The ORR was $53 \%$ with $20 \%$ of CR [9]. Nivolumab and pembrolizumab are the first two US Food and Drug Administration-approved mAbs targeting PD-1 for HL and provide promising results in the relapsed/refractory setting $[2,3]$.

An interesting area of ongoing research is the possibility of combining different immune checkpoint inhibitors given concomitantly or sequentially in the attempt to maximize the patient responsiveness. Nivolumab and pembrolizumab work by interfering with interaction between PD-1 or programmed death ligand-1 (PD-L1), thus they could represent two valuable and interchangeable alternatives to target relapsed/refractory HL [14]. In addition, the persistence of target on tumor microenvironment supports the rational for re-treatment. However, It is still to be understood when and where it is best to enter them in the relapsed/refractory HL therapeutic algorithm. In addition, due to the reported risks of early immune toxicity, more mature data are awaited to draw conclusions on safety of alloSCT after checkpoint inhibitors even useful preliminary information are available [15].

\section{Conclusion}

This is the first report which witnesses the safety and the antitumor activity of interchangeable anti-PD1 mAbs administered as a retreatment option and as a bridge to alloSCT in a patient who previously got an objective response to another anti-PD1 which brought her to ASCT.

\section{References}

1. Alinari L, Blum KA (2016) How I treat relapsed classical Hodgkin lymphoma after autologous stem cell transplant. Blood 127: 287-295.

2. Bond DA, Alinari $L$ (2017) Emerging treatment options for the management of Hodgkin's lymphoma: Clinical utility of nivolumab. J Blood Med 8: 41-54.

3. Maly J, Alinari L (2016) Pembrolizumab in classical Hodgkin's lymphoma. Eur J Haematol 97: 219-227.

4. Moskowitz AJ, Hamlin PA Jr, Perales MA, Gerecitano J, Horwitz SM, et al. (2013) Phase II study of bendamustine in relapsed and refractory Hodgkin lymphoma. J Clin Oncol 31: 456-460.

5. Younes A, Gopal AK, Smith SE, Ansell SM, Rosenblatt JD, et al. (2012) Results of a pivotal phase II study of brentuximab vedotin for patients with relapsed or refractory Hodgkin's lymphoma. J Clin Oncol 30: 2183-2189.

6. Kharfan-Dabaja MA, Hamadani M, Sibai H, Savani BN (2014) Managing Hodgkin lymphoma relapsing after autologous hematopoietic cell transplantation: A notso-good cancer after all. Bone Marrow Transplant 49: 599-606.

7. Green MR, Monti S, Rodig SJ, Juszczynski P, Currie T, et al. (2010) Integrative analysis reveals selective 9p24.1 amplification, increased PD-1 ligand expression, and further induction via JAK2 in nodular scleros-ing Hodgkin lymphoma and primary mediastinal large B-cell lymphoma. Blood 116: 32683277.

8. Green MR, Rodig S, Juszczynski P, Ouyang J, Sinha P, et al. (2012) Constitutive AP-1 activity and EBV infection induce PD-L1 in Hodgkin lymphomas and posttransplant lymphoproliferative disorders: Implications for targeted therapy. Clin Cancer Res 18: 1611-1618.

9. Chen R, Zinzani PL, Fanale MA, Armand P, Johnson NA, et al. (2017) Phase II study of the efficacy and safety of Pembrolizumab for relapsed/refractory classic Hodgkin lymphoma. J Clin Oncol 35: 2125-2132.

10. Matsuki E, Younes A (2016) Checkpoint inhibitors and other immune therapies for Hodgkin and Non-Hodgkin lymphoma. Curr Treat Options Oncol 17: 31.

11. Younes A, Ansell S (2016) Novel agents in the treatment of Hodgkin lymphoma: Biological basis and clinical results. Semin Haematol 53: 186-189.

12. Dodero A, Crocchiolo R, Patriarca F, Miceli R, Castagna L, et al. (2010) Pretransplantation [18-F]fluorodeoxyglucose positron emission tomography scan predicts outcome in patients with recurrent Hodgkin lymphoma or aggressive non-Hodgkin lymphoma undergoing reduced-intensity conditioning followed by allogeneic stem cell transplantation. Cancer 116: 5001-5011.

13. Younes A, Santoro A, Shipp M, Zinzani PL, Timmerman JM, et al. (2016) Nivolumab for classical Hodgkin's lymphoma after failure of both autologous stem-cell transplantation and bentuximab vedotin: A multicentre, multicohort, single arm phase 2 trial. Lancet Oncol 17: 1283-1294. 
Citation: Pellegrini C, Casadei B, Cellini C, Argnani L, Cavo M, et al. (2018) Sequential Double Bridging to Transplant with Diversified Anti-PD1 Monoclonal Antibodies Retreatment in Relapsed Hodgkin Lymphoma: A Case Report. J Cancer Sci Ther 10: 149-151. doi: 10.4172/19485956.1000534

14. Prasad V, Kaestner V (2017) Nivolumab and Pembrolizumab: Monoclonal antibodies against programmed cell death-1 (PD-1) that are interchangeable. Semin Oncol 44: 132-135.
15. Merryman RW, Kim HT, Zinzani PL, Carlo-Stella C, Ansell SM, et al. (2017) Safety and efficacy of allogeneic hematopoietic stem cell transplant after PD-1 blockade in relapsed/refractory lymphoma. Blood 129: 1380-1388. 